Z Rheumatol 2010 $69: 863$

DOI 10.1007/s00393-010-0678-1

๑) Springer-Verlag 2010

E. Märker-Hermann ${ }^{1} \cdot$ R.E. Schmidt ${ }^{2}$

${ }^{1}$ Klinik Innere Medizin IV (Rheumatologie, klinische Immunologie und Nephrologie), HSK Dr. Horst Schmidt Klinik GmbH, Wiesbaden

${ }^{2}$ Klinik für Immunologie und Rheumatologie, Medizinische Hochschule Hannover

\title{
Infektionen - Ursachen, Auslöser und Komplikationen rheumatischer Erkrankungen
}

chungen bei HLA-B27-assoziierter Chlamydien- und Yersinien-induzierter reaktiver Arthritis haben die reaktive Arthritis zur pathogenetischen „Modellerkrankung" der Spondyloarthritiden gemacht. M. Rihl und J. Kuipers führen in ihrem Beitrag in dieser Ausgabe der Zeitschrift für Rheumatologie aus, welche neuen pathogenetischen und diagnostischen Aspekte sich bei der reaktiven Arthritis in den letzten Jahren entwickelt haben und welche Perspektiven sich für die Therapie ergeben.

Viren können über die direkte Infektion synovialer Zellen über lytische Effekte, Immunkomplexbildung oder Induktion proinflammatorischer Zytokine zur Arthritis führen; alternativ, die vorgenannten direkten Viruseffekte aber nicht ausschließend, könnten Autoimmunreaktionen über molekulare Mimikry, „Bystander-Aktivierung“ oder „Epitope Spreading “ induziert werden. Virale Arthritiden stellen eine wichtige Differenzialdiagnose bei akuter oder subakuter Polyarthritis dar. Neben bekannten Viren wie Parvovirus B19, Röteln- oder Hepatitisviren haben im Rahmen des zunehmenden Ferntourismus auch in Europa durch Mückenstiche übertragene Alphaviren und die hierdurch ausgelösten akuten Erkrankungen mit Fieber, Exanthem und Polyarthritis an Bedeutung zugenommen. Nicht selten ist der Allgemeininternist oder der Rheumatologe neben tropenmedizinischen Kliniken mit diesen Patienten primär konfrontiert.
Schließlich sind gerade in den vergangenen Jahren mit der konsequenten immunsuppressiven Therapie immunologischer Systemerkrankungen und mit Einführung der Biologika in die Behandlung entzündlich-rheumatischer Erkrankungen Infektionen als Komplikation der Therapie rheumatischer Erkrankung in den Fokus unseres Interesses geraten. Die vorliegende Ausgabe der Zeitschrift für Rheumatologie legt daher in besonderer Weise einen Schwerpunkt auf die infektiösen Komplikationen antirheumatischer Therapien, deren Diagnostik und Prävention.

Ihre

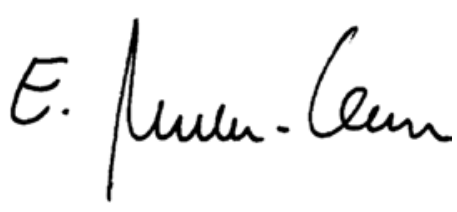

Elisabeth Märker-Hermann

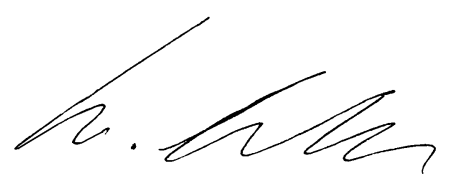

Reinhold E. Schmidt

\section{Korrespondenzadresse}

Prof. Dr. E. Märker-Hermann

Klinik Innere Medizin IV

(Rheumatologie, klinische Immunologie und Nephrologie),

HSK Dr. Horst Schmidt Klinik GmbH Ludwig Erhard Str. 100, 65199 Wiesbaden

Elisabeth.Maerker-Hermann@

HSK-Wiesbaden.de intensiv erforscht. Gerade Erkenntnisse aus ex vivo immunologischen Untersu- 